\title{
Neurotheologie - empirische Forschung an der Nahtstelle zwischen Wissen und Glauben
}

\author{
Nietzsche bezeichnete den Menschen als «hoffnungslos religiös». C. G. Jung konsta- \\ tierte, die menschliche Seele sei «naturaliter religiosa». Tatsächlich ist die grosse \\ Mehrheit der Erdbevölkerung in irgendeiner Weise gläubig. Der militante Atheist \\ R. Dawkins macht dafür vor allem die Indoktrination durch Eltern und andere Erzie- \\ hungspersonen verantwortlich. Ohne der Tradition ihren Stellenwert absprechen zu \\ wollen, sucht der Beitrag nach den Grundlagen der Religiosität im Sinne einer lern- \\ fähigen Hirnfunktion.
}

\section{Thomas Knecht}

Lesen Sie hierzu auch den Kommentar von Jürg Kesselring auf Seite 787 .
Korrespondenz:

Dr. med. Thomas Knecht Leitender Arzt

Psychiatrische Klinik Postfach 154

CH-8596 Münsterlingen

Tel. 0716864028

Fax 0716864035

thomas.knecht[at]stgag.ch

\section{Einleitung}

«Religion» ist ein breit angelegtes und überaus facettenreiches Konzept. Eine verkürzte Lexikon-Definition bringt in etwa Folgendes zum Ausdruck: «Vom Menschen ausgehende geistig-seelische Hinwendung auf einen letzten Seinsgrund, vielgestaltige Antwort des Menschen auf die Erfahrung einer machtvollen überirdischen Existenz».

Es war indessen seit jeher klar, dass zwischen der Religio als kulturell geschaffenem Glaubenssystem mit seinen mannigfachen Inhalten und der Religiositas, der grundlegenden Glaubensfähigkeit der Menschenpsyche, unterschieden werden muss. Nur die Letztere kann Gegenstand von naturwissenschaftlichen Untersuchungen sein, zumal heute diverse Befunde vorliegen, die dafür sprechen, dass es sich bei der Glaubensfunktion um eine evolutionär entwickelte Besonderheit des menschlichen Gehirns handelt.

\section{Befundlage}

Aus der Vielzahl der klinischen und experimentellen Befunde, die im Zusammenhang mit religiösem Erleben erhoben wurden, seien im Folgenden die wichtigsten kurz resümiert:

Bemerkenswert sind die Untersuchungsergebnisse von Ramachandran [1]: Dieser Neurowissenschaftler aus San Diego ging von der Erfahrungstatsache aus, dass Temporallappen-Epileptiker oftmals ein verstärktes religiöses Empfinden haben, und so verglich er sie testmässig mit gesunden Probanden, indem er beiden Gruppen auf einem Monitor Wörter mit neutralem, sexuellem oder religiösem Inhalt darbot. Anhand der Schwankungen der Hautleitungsfähigkeit bestimmte er dann die Reaktionsstärke der Probanden auf diese Reizworte. Dabei zeigte sich, dass die Gesunden die heftigsten Reaktionen auf die sexuellen Wörter zeigten, während die Epileptiker auf die religiösen Wörter am intensivsten reagierten. In einem weiteren Schritt lokalisierte Ra- machandran mittels Positronenemissionstomographie (PET) denjenigen Ort im Schläfenlappen, der die höchste Mehraktivierung bei religiösen Reizwörtern zeigte. Dieser ging sodann als God spot in die Laienpresse ein.

Ein grosses Echo riefen auch die SPECT-Experimente von Newberg und D'Aquili [2] hervor. Diese Autoren, ein Radiologe und ein Psychiater, gingen in ihrer Untersuchung der Frage nach, ob spirituelle Erfahrungen wie Trance und Meditation weltweit mit gleichartigen Hirnaktivitätsmustern einhergehen. Ihre Probanden waren buddhistische Mönche sowie christliche Nonnen. Es zeigte sich, dass sich - unabhängig vom Glaubensbekenntnis - völlig gleichartige Aktivitätsverschiebungen ergaben. So nahmen Durchblutung und Sauerstoffverbrauch im oberen posterioren Parietallappen drastisch ab. Diese Region bildet bekanntlich ein Orientierungsfeld, das den Menschen in Raum und Zeit verortet; eine Funktionsverminderung ist demnach mit einem Erlebnis des Entrücktseins gut vereinbar. Daneben nahm die Frontalhirnaktivität markant zu, was ebenfalls zum hochkonzentrierten meditativen Zustand passt, zumal dies u. a. der Sitz der Aufmerksamkeitslenkung ist.

Am meisten Aufsehen - aber auch Kontroversen erregten wohl die Versuche des kanadischen Neurologen Michael Persinger [3]: Dieser bediente sich der transkraniellen Magnetstimulation, indem er einen Motorradhelm mit acht Magnetspulen versah, welche die Gehirne seiner Probanden an umschriebener Stelle stimulieren sollten. Tatsächlich gaben in der Folge rund 80\% der Versuchspersonen mystische Erfahrungen an: Entweder erlebten sie die Präsenz Gottes, allenfalls eines Schutzengels, oder aber - falls sie Atheisten waren - eine Art Unio mystica mit dem Universum. Nach Persingers Auffassung war damit der «Gottesmodul» entdeckt. Allerdings liessen sich seine Ergebnisse in ähnlichen Versuchssettings nicht ohne weiteres replizieren: Granqvist et al. [4] 


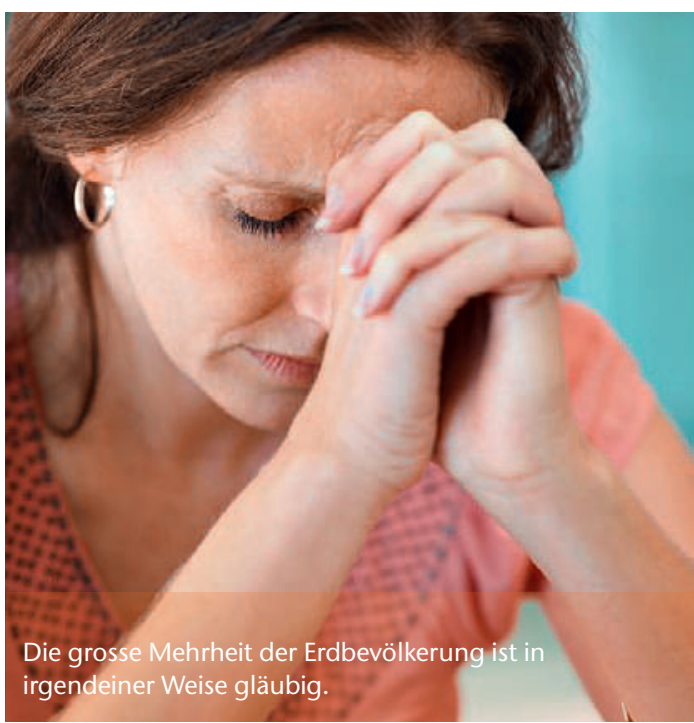

arbeiteten ebenfalls mit dem «Gotteshelm», setzten diesen aber zu Kontrollzwecken nur bei der Hälfte ihrer Probanden unter Strom. Es zeigte sich nun aber, dass auch ein Teil der nur scheinbar Stimulierten entsprechende mystische Erfahrungen berichteten, was natürlich die Frage eines Plazeboeffektes aufwirft. Allerdings musste sich auch Granqvist Kritik bezüglich seines methodischen Vorgehens gefallen lassen.

Von anderer Seite [5] wurde ausserdem darauf hingewiesen, dass bei solchen Erfahrungen nicht nur der temporale God spot aktiv wird, sondern ein ganzes neuronales Netzwerk, das $\mathrm{u}$. a. auch frontale und occipitale Areale (visueller Cortex) einschliesst.

Des Weiteren wurden klinische Untersuchungen an Parkinsonpatienten gemacht, die bekanntlich einen frontalen Dopaminmangel aufweisen. Im Gegensatz zu Schizophrenen, deren frontale dopaminerge Neurotransmission hyperaktiv ist und die nicht selten unter extremen religiösen Wahnideen leiden, war bei den Parkinsonkranken die Religiosität vermindert [6].

In eine ähnliche Richtung weist das Marsh-ChapelExperiment von Pahnke [7], der bei einer Gruppe von Theologiestudenten das Halluzinogen Psilocybin (30 mg) applizierte und sie danach an einer Karfreitagsliturgie teilnehmen liess, um auf diesem Wege mystische Erfahrungen hervorzurufen.

Schliesslich wurde sogar auf Ebene der DNA nach dem «Gottes-Gen» gesucht, dies ausgehend von der Beobachtung, dass eine hochgradige Religiosität in bestimmten Familien stark gehäuft vorkommt. Dies kann, muss aber nicht nur durch Milieufaktoren bedingt sein. Dean Hamer [8] machte die Probe aufs Exempel und stellte mit der «SelbstTranszendenz-Skala» von R. Cloninger eine Gruppe von tiefreligiösen Testpersonen zusammen. Bei breitangelegten genetischen Analysen stellte er in der Folge fest, dass diese Menschen im Vergleich zu Kontrollpersonen eine spezielle Variante des VMAT 2-Gens auf Chromosom 10 aufwiesen, wobei an umschriebe- ner Stelle (Nucleotid-Position 33,050) Adenin durch Cytosin ersetzt war. VMAT 2 (= vesicular monoamine transporter) ist ein Protein, das massgeblich an der Bereitstellung von Monoaminen wie Dopamin und Serotonin in der Synapse und damit an der Regulation von Grundstimmung und Emotionalität (inkl. Zuversicht, Urvertrauen u. ä.) beteiligt ist. Allerdings musste Hamer einräumen, dass nur 40-50\% der beobachteten Unterschiede betr. Selbst-Transzendenz auf diesen genetischen Faktor zurückzuführen waren. Womit er die bekannte Problematik des Schliessens von punktuellen Korrelationen auf umfassende Kausalitäten aufzeigte.

\section{Religiositas als Evolutionsprodukt?}

Die oben resümierte Befundlage liefert in der Tat Anhaltspunkte dafür, dass Religiosität eng an das menschliche Gehirn gebunden ist. Dennoch ist vor einer Überinterpretation dieser Ergebnisse zu warnen. Eine präzise Lokalisation des religiösen Glaubens in einem eng umschriebenen Hirnareal kann daraus sicher nicht abgeleitet werden. $\mathrm{Zu}$ eng gesteckt sind derzeit noch die Erkenntnisgrenzen, zu gering ist die Konsistenz des Befundmaterials aus diesen klinischen Experimenten.

Es scheint indessen legitim, die Frage aufzuwerfen, wie sich die Menschheit im Zuge ihrer Entwicklungsgeschichte diese in der Natur einzigartige Fähigkeit der Religiosität aneignen konnte und wieso sie dieses tat. Damit sind natürlich evolutionäre Erklärungsmodelle angesprochen, die in Psychologie und Psychiatrie eine zunehmend wichtigere Rolle spielen [9]. Letztlich geht es dabei um die Frage, ob und inwieweit die Religiosität im weitesten Sinne einen Überlebensvorteil im Zuge der Hominidenevolution dargestellt haben könnte. Wie Voland [10] treffend festhält, müsste sie zumindest drei Kriterien erfüllen, um als entwicklungsgeschichtliche Anpassung zu gelten:

1. Sie müsste vererbt sein.

2. Sie müsste Produkt eines historischen Ausleseprozesses sein, d. h. ein zweckgerichtetes Design aufweisen.

3. Sie müsste ein Adaptationsproblem der betreffenden Spezies lösen.

Es ist nun festzuhalten, dass zentrale Elemente der Religiosität tatsächlich diese Kriterien erfüllen, so z. B. die Fähigkeit, verschiedenartige Bewusstseinszustände zu erlangen, aber auch das Bonding, d.h. die fördernden Effekte auf Gruppenbildung sowie Kommunikation, somit auch auf die persönliche Identitätsbildung, die stets wesentlich durch die Gruppenzugehörigkeit bestimmt war.

Es ist aber zu betonen, dass weder die Glaubensformen (Ein- oder Mehrgottglaube) noch Dogmen, Bilderkanones mit Heiligen, Engeln und Dämonen, geschweige denn Rituale und Sakramente als evolutionäre Anpassungen im Darwinschen Sinne zu sehen sind. Diese Phänomene sind durchwegs kulturelle Schöpfungen des menschlichen Bewusstseins, 
die allerdings eine Reihe evolutionärer Anpassungen der kognitiven Strukturen zur Voraussetzung hatten.

Was die Adaptationsmerkmale im Einzelnen angeht, so darf heute festgehalten werden, dass die oben angeführten Punkte 1-3 in ihrer Tatsächlichkeit weitgehend empirisch bestätigt sind: So sprechen einzelne Studien doch dafür, dass die grundsätzliche Bereitschaft, religiöse Inhalte zu glauben, zumindest durch genetische Faktoren mitbestimmt ist [z. B. 11], wie das mittlerweile auch für andere essentielle Wesenszüge wie Intelligenz, Narzissmus, Dissozialität oder Neurotizismus belegt ist.

Der funktionale Anpassungswert der Religiosität kommt dagegen v. a. im Kontext der Grossgruppenbildung zum Ausdruck. Gemeinsame Glaubensvorstellungen, die mit diversen Ritualen gekoppelt sind, ermöglichen eine bessere Koordination und Kooperation (vgl. Gebote und Verbote) unter den Mitgliedern, was diesen Verbänden einen Vorteil im Wettbewerb der Gruppen verschaffte. Somit ermöglichten religiös verstärkte Identitätsbildung und Solidarität eine Expansion dieser Völker. Dabei darf es als erwiesen gelten, dass Personen von ausgeprägter Religiosität fertiler sind als solche ohne religiöse Bindung [Übersicht bei 10]. Schliesslich trägt die Religiosität im Verbund mit den auf ihr fussenden Religionen dazu bei, dass der Mensch von einer anderen evolutionär entwickelten Fähigkeit, nämlich von Täuschung und Betrug, zurückhaltender Gebrauch macht, zumal jede Religion auch eine Auswahl von «Ehrlichkeitssignalen» liefert: Schwüre, Gelöbnisse, Ehezeremonien usw.

Die allwissenden, metaphysischen Wesenheiten hatten dabei die Funktion, auch das verdeckte Treiben der Gläubigen zu überwachen und nötigenfalls $\mathrm{zu}$ ahnden, was eine strenge Normenkontrolle gewährleistete. Roes u. Raymond [12] wiesen nach, dass der Glaube an einen strafenden Gott mit der Grösse (und damit Unübersichtlichkeit) einer Population zunimmt. Daraus lässt sich ersehen, dass zumindest unter ursprünglichen Lebensverhältnissen die Zugehörigkeit eines Individuums zu einer religiösen Gruppe mit Sicherheit seine Überlebens- und Fortpflanzungschancen verbesserte.

Vom Standpunkt der Gruppe aus ergab sich aufgrund dieser verbesserten, soziokulturell bedingten Kohäsion als Wettbewerbsvorteil die Möglichkeit eines zahlenmässigen Wachstums. Rein von den sozialkognitiven Fähigkeiten resp. der Gehirngrösse des Menschen her wäre die Gruppengrösse gemäss der Dunbarschen Zahl auf rund 150 Mitglieder beschränkt geblieben [13].

So schufen die (Stammes-)Religionen einen sozialen Kitt, der eine deutliche Grössenzunahme der sozialen Verbände zuliess. Damit kam es zu einer Überlegenheit der entsprechend ausgestatteten Gemeinschaften gegenüber kleineren Gruppen, die sie nun erfolgreich verdrängen oder unterwerfen konnten (Gruppenselektion).
Erfolgreiche Religionen waren demnach jene, welche von ihren Glaubensvorstellungen her «mehrheitsfähig» waren, d. h. durch das Angebot eines gemeinsamen spirituellen Nenners möglichst grosse Zahlen von Anhängern unter ihrem Banner vereinigen konnten.

Auch wenn diese Befunde und Überlegungen zum Teil sicher noch vorläufigen Charakter haben, darf mit der gebotenen Vorsicht konkludiert werden, dass Glaubensinhalte zwar kulturelle Schöpfungen sind, die in jedem Kulturraum ihre eigene Entwicklung durchliefen, dass aber die elementare Glaubensfunktion eine evolutionär angelegte, dem menschlichen Gehirn universal inhärente Fähigkeit ist, welche immer und überall auf zeit- und kulturgemässe Art sozialisiert resp. indoktriniert wurde.

\section{Literatur}

1 Ramachandran VS, Blakeslee S. Phantoms in the brain. New York: William Morrow; 1998.

2 Newberg AB, Pourdehnad M, Alavi A, D'Aquili E. Cerebral bloodflow during meditative prayer. Percept Mot Skills. 2003;97:625-30.

3 Persinger MA. Experimental stimulation of the god experience, In: Joseph R (ed.): Neurotheology. San José: University Press California; 2003.

4 Granqvist P, Fredrikson M, Unge P, Hagenfeldt A, Valind S, Larhammer D, Larsson M. Sensed presence and mystical experiences are predicted by suggestibility, not by the application of weak complex transcranial magnetic fields. Neuroscience Letter, 2005;379: $1-6$.

5 Beauregard M, Paquette V. EEG activity in Carmelite nuns during a mystical experience. Neuroscience Letters. 2008;444:1-4.

6 Harris E, Mc Namara P. Neurologic constraints in evolutionary theories of religion. In: Voland E, Schiefenhövel W (eds). The biological evolution of religious mind and behavior. Berlin: Springer; 2009. S. 205-215

7 Pahnke WN. Drugs and mysticism: An analysis of the relationship between psychedelic drugs and mystical consciousness. Harvard University: Unpublished doctoral dissertation; 1963.

8 Hamer D: Das Gottes-Gen. Warum uns der Glauben im Blut liegt. München: Kösel; 2006

9 Brüne M. Ribbert H. Evolutionsbiologische Konzepte in der Psychiatrie. Frankfurt a. M.: Lang; 2001.

10 Voland E. Evaluating the evolutionary status of religiosity and religiousness. In: Voland E, Schiefenhövel W (eds). The biological evolution of religious mind and behavior. Berlin: Springer 2009; S. 9-24.

11 Bouchard TJ. Authoritarianism, religiousness, and conservativism: Is «obedience to authority» the explanation for their clustering, universality and evolution? In: Voland E, Schiefenhövel W (eds): The biological evolution of religious mind and behavior. Berlin: Springer; 2009. S. 165-80.

12 Roes FL, Raymond M. Belief in moralizing gods. Evolution and Human Behavior. 2003;24:126-35.

13 Dunbar RIM. The social brain: mind, language, and society in evolutionary perspective. Annual Review of Anthropology. 2003;32:163-81. 